\title{
A case of neonatal alloimmune thrombocytopenia from human platelet antigen $\mathbf{5 b}$ incompatibility
}

\author{
Takehiro Serikawa $^{a}$ Yasuhiro Takahashia Kaya Ichikawa ${ }^{a}$ Ruriko Uemura ${ }^{a}$

 \\ Kenichi Tanaka ${ }^{a}$ \\ ${ }^{a}$ Department of Obstetrics and Gynecology, and ${ }^{\mathrm{b}}$ Department of Pediatrics, Niigata University \\ Medical and Dental Hospital, Niigata, Japan
}

1-757 Asahimachi-dori, Chuo-ku, Niigata City, 951 -8510, Niigata Prefecture, Japan

\section{Keywords:}

alloimmune thrombocytopenia, humanspecific platelet antigen, amniocentesis, mixed passive hemagglutination

\begin{abstract}
Anti-human platelet-specific antigen (HPA) antibody often causes neonatal alloimmune thrombocytopenia (NAIT). The antibody is produced due to the feto-maternal transfusion of incompatible platelets. In this case study, antiHPA-5b was detected in the serum of a 30-year-old female patient. Using blood or amniotic fluid, the patient' s HPA-5 phenotype was determined to be $a+b-$, whereas those of the husband, son and fetus were $a+b+$. From these findings, we concluded that there was an incompatibility of maternal and fetal HPA. Cordocentesis was performed at 34 weeks of gestation and the fetal platelet count was sufficient for vaginal delivery. A transfusion of HPAmatched platelet was prepared. The baby was delivered by vaginal delivery and there were no physical signs of thrombocytopenia.
\end{abstract}

\section{Case report}

A 30-year-old Japanese woman with gravida 2, para 1, gave birth to a female neonate by vaginal birth at 40 weeks of gestation. Two years before, she gave birth to a male neonate with IUGR and congenital duodenal atresia, whose platelet count at the time of birth was 219

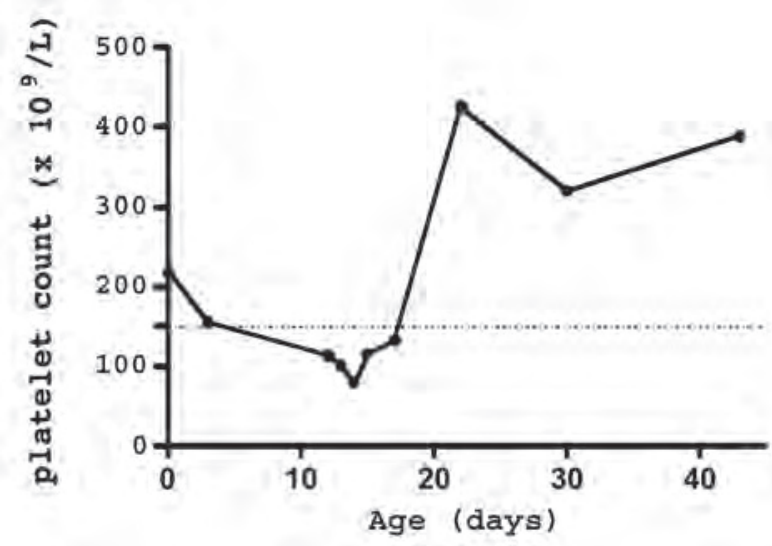

Fig. 1 Clinical course of the patient's previous child. Initial platelet count was $219 \times 10^{9} / \mathrm{L}$. Platelet count at day 14 dropped to $79 \times 10^{9} / \mathrm{L}$. Without any treatment, platelet count normalized by day 22 . $\times 10^{9} / \mathrm{L}$. His platelet count dropped transiently to $79 \times$ $10^{9} / \mathrm{L}$ on day 14 , but returned to $425 \times 10^{9} / \mathrm{L}$ on day 22 without any treatment (Fig. 1). No intracranial hemorrhage, purpura, or petechiae were noted. During this previous pregnancy, the mother's platelet count was $119 \times 10^{9} / \mathrm{L}$ at 38 weeks of gestation and increased

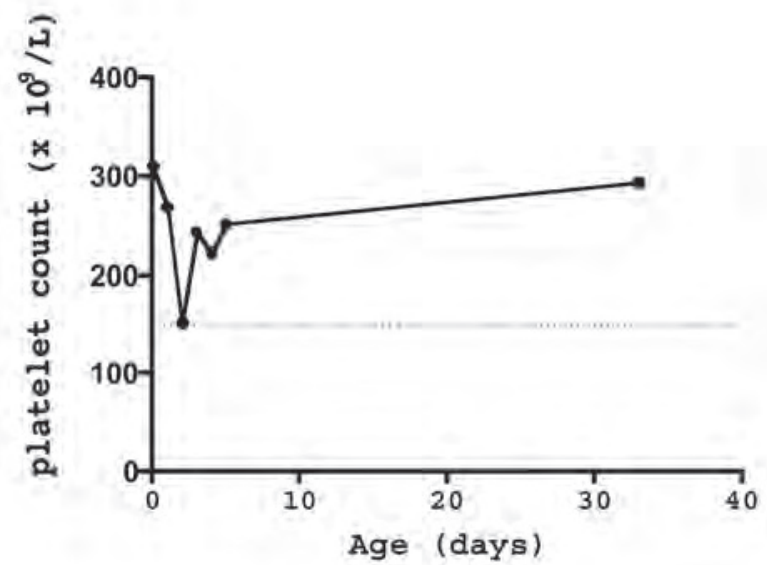

Fig.2 Clinical course of the daughter. Initial platelet count was $310 \times 10^{9} / \mathrm{L}$. Platelet count at day 2 dropped to $151 \times$ $10^{9} / \mathrm{L}$. Without any treatment, platelet count normalized by day 3.

Reproductive Immunology and Biology Volume24 • Number 1 • June2009 Japan Society for Immunology of Reproduction 
Table 1 HPA type (MPHA method) of the patient, husband,son and fetus

\begin{tabular}{lccccccc}
\hline & $2 \mathrm{~b}$ & $3 \mathrm{a}$ & $4 \mathrm{a}$ & $4 \mathrm{~b}$ & $5 \mathrm{a}$ & $5 \mathrm{~b}$ & $6 \mathrm{~b}$ \\
\hline patient & + & + & + & - & + & - & - \\
husband & + & - & + & - & + & + & - \\
son & + & + & + & - & + & + & - \\
fetus & + & + & + & - & + & + & - \\
\hline
\end{tabular}

The platelet phenotype of the patient was HPA-5 (a+b-) while the husband and children platelet phenotypes were HPA-5 $(a+b+)$.

to $217 \times 10^{9} / \mathrm{L}$ on day 14 after delivery.

The patient's platelet count was 140 and $141 \times 10^{9} / \mathrm{L}$ at 8 and 14 weeks of gestation, respectively. A plateletspecific antibody, anti-HPA-5b, was detected with a titer of 1:2048 in her serum, using the mixed passive hemagglutination (MPHA) method, suggesting that the former child's thrombocytopenia might have been due to neonatal alloimmune thrombocytopenia (NAIT). Platelet genotyping of the patient, her husband, and her son gave a result of HPA-5 $(a+b-)$, HPA-5 $(a+b+)$ and HPA-5 $(a+b+)$ (Table 1). Because no blood transfusion was given, the patient could have been sensitized during the previous pregnancy through her son; we diagnosed that the son had neonatal alloimmune thrombocytopenia due to the anti-HPA-5b antibody. Since the fetus also had a $50 \%$ risk of NAIT, amniocentesis for fetal genotyping was performed at 28 weeks of gestation. The platelet genotyping of the fetus was HPA-5 $(a+b+)$ using amniotic fluid (Table 1). Since the symptoms of NAIT were more severe for the fetus than for the former child, cordocentesis was performed at 34 weeks of gestation to count the fetal platelet and decide the delivery mode. The platelet count was $203 \times$ $10^{9} / \mathrm{L}$ and hemoglobin was $117 \mathrm{~g} / \mathrm{L}$. Peak systolic velocity in the middle cerebral artery of the fetus was measured up to delivery and no evidence of fetal anemia was noted. After vaginal birth, the platelet count of the female neonate was $310 \times 10^{9} / \mathrm{L}$. The girl had no purpura or petechiae, so HPA-matched platelet transfusion was not attempted. The platelet count of the neonate dropped to $151 \times 10^{9} / \mathrm{L}$ on day 2 , but retuned to $243 \times 10^{9} / \mathrm{L}$ on day 3 without any treatment (Fig. 2). Anti-HPA-5b was detected in the umbilical cord blood.

\section{Discussion}

The incidence of anti-HPA antibody is $0.6-0.9 \%$ in pregnant women $[1,2]$.

NAIT occurs when the maternal antibodies of an immunized antigen-negative mother cross the placenta and cause destruction of sensitized fetal platelets [3]. NAIT recurs in 70 to 90 percent of subsequent pregnancies, is often severe, and usually develops earlier with each successive pregnancy [4].

Furthermore, severe thrombocytopenia places the baby at risk for intracranial hemorrhage and other bleeding complications [5].

Due to the former birth of an affected child, an occurrence of NAIT was suspected in this case. Using the MPHA method, anti-HPA-5b was strongly detected with a titer of 1:2048 in the patient's serum. Platelet genotyping of the patient, her husband, and her son gave a result of HPA-5 $(\mathrm{a}+\mathrm{b}-)$, HPA- $5(\mathrm{a}+\mathrm{b}+)$, and HPA- 5 $(a+b+)$, confirming that the son's disease was NAIT due to the incompatibility of platelet antigen. The patient received no transfusion, so the HPA-5b antigen could have been sensitized by feto-maternal transfusion during the previous pregnancy. Since the fetus had a $50 \%$ risk of NAIT because the husband's genotype was heterozygous, an amniocentesis, which is less invasive, was performed for DNA typing at 28 weeks of gestation after informed consent was obtained. The result was HPA-5 $(a+b+)$ and it was diagnosed that there was fetomaternal incompatibility of HPA-5b.

For "standard risk" patients, who are defined as women with documented alloimmune thrombocytopenia who did not deliver an infant with an intracranial hemorrhage in a prior pregnancy [6], it is recommended that vaginal delivery be allowed only for patients whose fetuses have a platelet count greater than $100 \times 10^{9} / \mathrm{L}$ 
at 32 weeks of gestation. In this case, the neonate' $\mathrm{s}$ HPA-5b alloimmunization had substantially less severe manifestations than other HPA-antigen alloimmunizations; the reported incidence of intracranial hemorrhage due to HPA-5b alloimmunization is $0-8 \%$ [7]. However, due to the risk of occurrence of NAIT, cordocentesis was performed at 34 weeks of gestation in order to decide the mode of delivery. The platelet count was sufficient for vaginal delivery. The fetus was delivered by vaginal birth, and there were no bleeding complications. The fetal platelet count transiently decreased, but subsequently increased without any treatment.

There must be a 50\% risk of feto-maternal incompatibility of HPA-5b about next pregnancy because the patient has anti-HPA-5b antibody and her husband has a HPA-5 (a+b+) antigen. Recently, maternally administrated intravenous immunoglobulin has been the most successful therapy [6] however health insurance adaptation cannot be accepted in our country. In any case, a sufficient counseling about a recurrence of NAIT is important.

\section{Acknowledgement}

We would like to thank Dr. Hironori Kobayashi, Dr. Shoji Morita and Dr. Susumu Inoue (Japanese Red Cross Saitama Blood Center) for HPA typing.

\section{References}

1. Enomoto T, Maruoka H, Hanagaki S, Morita S, Shimamura M, Hando K, Ishijima A, Yoshioka A, Yuasa S, Takahashi K, Ohto H. 2000. Pregnancy- induced alloimmunization against platelet antigens: HLA and human platelet antigens (HPA). Jpn J Transfus Med. 46: 467-473.

2. Nagao N, Taniue A, Tomita N, Tomita T, Okubo Y, Ban C, Shibata Y. 1998. Neonatal alloimmune thrombocytopenia due to anti-HPA and the incidence of HPA antibodies in pregnant Japanese women. Jpn J Transfus Med. 44: 317-321.

3. Ohto H, Miura S, Ariga H, Ishii T, Fujimori K, Morita S. 2004. The natural history of maternal immunization against foetal platelet alloantigens. Transfus Med. 14: 399-408.

4. Silver RM, Porter TF, Branch DW, Esplin MS, Scott JR. 2000. Neonatal alloimmune thrombocytopenia: antenatal management. Am J Obstet Gynecol. 182: 1233-1238.

5. Overton TG, Duncan KR, Jolly M, Letsky E, Fisk NM. 2002. Serial aggressive platelet transfusion for fetal alloimmune thrombocytopenia: platelet dynamics and perinatal outcome. Am J Obstet Gynecol. 186: 826-831.

6. Berkowitz RL, Lesser ML, McFarland JG, Wissert M, Primiani A, Hung Crystal, Bussel JB. 2007. Antepartum treatment without early cordocentesis for standard-risk alloimmune thrombocytopenia. Obstet Gynecol. 110: 249-255.

7. Paladini D, Maruotti GM, Sglavo G, Fratellanza G, Quarantelli M, Martinelli P. 2007. Massive fetal hemorrhage and fetomaternal alloimmune thrombocytopenia from human platelet antigen $5 b$ incompatibility: an unusual association. Ultrasound Obstet Gynecol. 29: 471-474. 